\title{
Congenital morphea profunda in a 7-year-old male patient with coexistence of fibrous hamartoma of infancy: a rare case report
}

\author{
Katarzyna A. Tomaszewska ${ }^{1,2}$, Joanna Krzysiek ${ }^{1}$, Aleksandra Kobusiewicz ${ }^{1,2}$, Zofia Gerlicz-Kowalczuk ${ }^{1,2}$, \\ Anna Zalewska-Janowska², Andrzej Kaszuba ${ }^{1}$ \\ ${ }^{1}$ Department of Dermatology, Pediatric Dermatology and Oncology, Medical University of Lodz, Lodz, Poland \\ ${ }^{2}$ Psychodermatology Department, Chair of Clinical Immunology and Microbiology, Medical University of Lodz, Lodz, Poland \\ Adv Dermatol Allergol 2018; XXXV (6): 638-640 \\ DOI: https://doi.org/10.5114/ada.2018.77617
}

Scleroderma is a rare spectrum of fibrosing disorders ranging from systemic sclerosis to localized scleroderma (morphea). The etiology is believed to involve activation of fibroblasts, increased production of collagen, and immunologic abnormalities [1]. The incidence of localized scleroderma is around 0.3 to 3 cases per 100000 inhabitants/ year. The prevalence of morphea is similar in children and adults. The peak incidence occurs in the fifth decade of life in adults, whereas $90 \%$ of children are diagnosed with the disease between 2 and 14 years of age [2].

Childhood scleroderma can be associated with significant morbidity and disability [3, 4]. Superficial patches of morphea may be relatively benign. However, linear scleroderma tends to involve not only the skin but also subcutaneous tissue, muscle tissue and bone, resulting in functional disabilities and cosmetic problems. Many children develop severe atrophy of the extremities, deformities, contractures and limb length discrepancies. In the en coup de sabre (ECDS) variety, involvement of the underlying structures may cause hemiatrophy of the face and facial deformity or even eye and brain complications [5, 6].

Two large international retrospective studies of childhood scleroderma published in 2006 highlighted the heterogeneous nature of scleroderma and juvenile systemic sclerosis [7, 8]. Like many other connective tissue diseases, juvenile localized scleroderma mainly involves females. This female preponderance is most marked after the age of 10 and is more evident in the generalized morphea and deep morphea subtype. In a cohort study conducted by Zulian et al., linear scleroderma (LS) was the most frequent subtype (65\%), followed by plaque morphea (PM) (26\%), generalized morphea (GM) (7\%) and deep morphea (DM) (2\%). Approximately $15 \%$ of the patients had a mixed subtype [7]. Of note, the most frequent form of localized scleroderma in adults is plaque morphea $[9,10]$.
We present a case of a 7-year-old male patient, who presented with atrophic plaques on the trunk and deformity of the right lower limb. Physical examination revealed multiple hyperpigmented ill-defined patches across the trunk. The biggest patch was localized in the left lower quadrant of the trunk and three other patches were on the chest. In addition, there were longitudinally arranged linear, band-like lesions extending the right lower limb with muscle atrophy and knee joint flexion contracture. The superficial blood vessels were visible within the lesions. The overlying skin was depressed, tightly adhered to the deep plane and difficult to pinch (Figure 1).

The symptoms were present since birth with gradual progression. Congenital lesions were described in detail by a neurology consultant in his first medical documentation. Systemic examination did not reveal any abnormality clinically, other than the limitations of movements due to contractures. There was no personal or family history of autoimmune, connective tissue disease or Raynaud's phenomenon. Hematological examination, blood biochemistry, and urine analysis results were normal. The antinuclear antibodies, rheumatoid factor, anti-Borrelia burgdorferi antibodies IgM and IgG were negative.

Skin biopsies demonstrated characteristic histopathological findings consistent with the diagnosis of morphea - the replacement of normal dermis and hypodermis structures by abnormal collagen (Figure 2). Based on the long lasting experience of our Department, procaine chelating properties and its beneficial effects on the microcirculation, the patient started injectable procaine benzylpenicillin with intravenous pentoxifylline infusion therapy with good outcome (skin thickness improvement from marked to moderate according to the Localized Scleroderma Skin Damage Index). The patient was also enrolled in a physical exercise program.

Address for correspondence: Katarzyna A. Tomaszewska MD, Department of Dermatology, Pediatric Dermatology and Oncology, Medical University of Lodz, 1/5 Kniaziewicza St, 90-435 Lodz, Poland, phone/fax: +48 4225161 92, e-mail: tomaszewska.katarzyna@o2.pl Received: 17.07.2017, accepted: 2.08.2017. 
Furthermore, the patient was diagnosed with fibrous hamartoma of infancy $(\mathrm{FHI})$ in his early infancy. The child had a history of several surgeries, including excision of the chest tumor at one month of age, excision of the right inguinal tumor at three months of age, excision of the tumor located on the back at 3 months of age. The patient underwent excision of the left calf tumor at the age of 6 years and right calf tumor at the age of 7. Magnetic resonance imaging was performed repeatedly. The indications for hormonal and cytostatic chemotherapy have not been established so far. The patient is under the care of the Dermatology, Neurology, Ophthalmology and Genetics Departments.

Fibrous hamartoma of infancy is a rare, benign tumor of the subcutis and lower dermis, which usually occurs within the first 2 years of life. Twenty-three percent are congenital. The histogenesis of $\mathrm{FHI}$ is unclear. The histology of lesion reveals a disorganized proliferation of tissues normal to the site in which they are found [11].

The tumor mainly involves males and is most commonly found in the axilla, shoulder, upper arm, inguinal region and chest wall; however, isolated cases have been reported involving the foot, scalp, perineal region, gluteal region, and scrotum. The prognosis of $\mathrm{FHI}$ is excellent with local surgical excision and it rarely recurs. Most re-

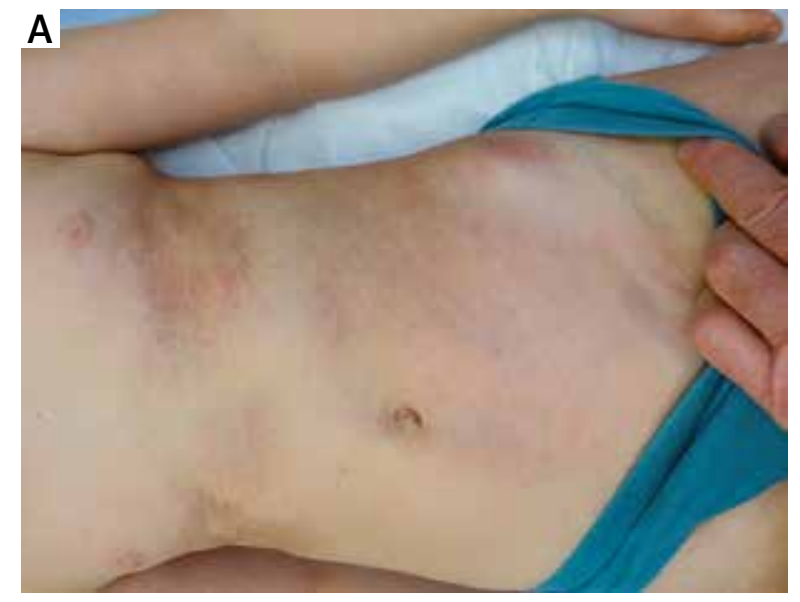

Figure 1. A, B - Multiple atrophic ill-defined patches across the trunk. C - Longitudinally arranged linear, band-like lesions extending to the right lower limb with muscle atrophy and knee joint flexion contracture ported cases have been of a single lesion, but rare cases of multiple lesions have been described [12].

The patient developed congenital, multiple lesions type of $\mathrm{FHI}$ and he still develops new lesions despite the fact of being 7 years old now.

Congenital morphea profunda is quite rare and probably is a quite highly underestimated condition in neonates, with only a few cases reported in the literature. According to the international study by Zulian in a group of 750 patients with juvenile localized scleroderma, 6 (0.8\%) patients had scleroderma-related lesions at birth [7]. All patients had linear scleroderma, in 4 cases involving the face with en coup de sabre appearance. Two patients were misdiagnosed as having the skin infection, one nevus, one salmon patch, and two undefined skin lesions. This study also reported a delay in diagnosis of up to 4 years [13]. Joshi et al. reported the first case of biopsy-confirmed congenital plaque morphea in a 4-year-old boy on the abdomen since birth [14]. A similar case was described by Castanon et al. in a 6-month-old girl [15]. Lipson et al. reported the case of congenital linear scleroderma en coup de sabre diagnosed in an adult following the initial misdiagnosis of birth trauma [16]. To the best of our knowledge, the congenital morphea profunda coexisting with fibrous hamartoma of infancy has not been reported yet. Despite numer-
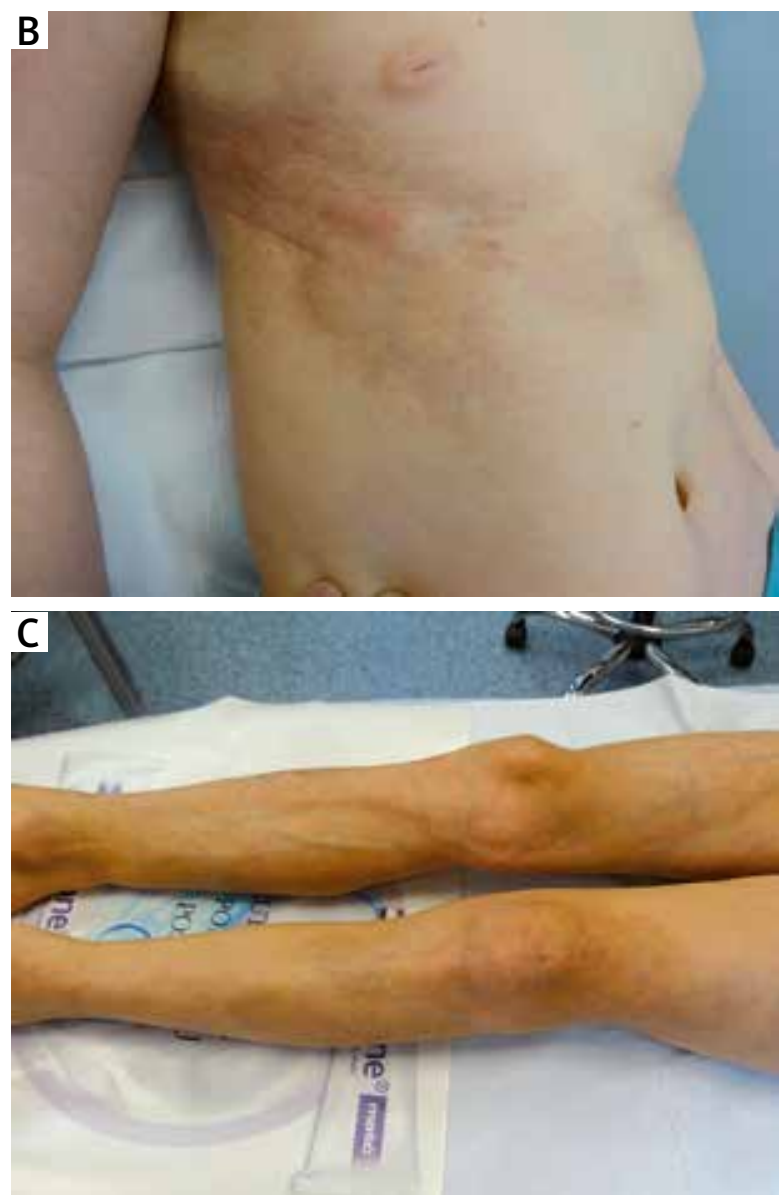

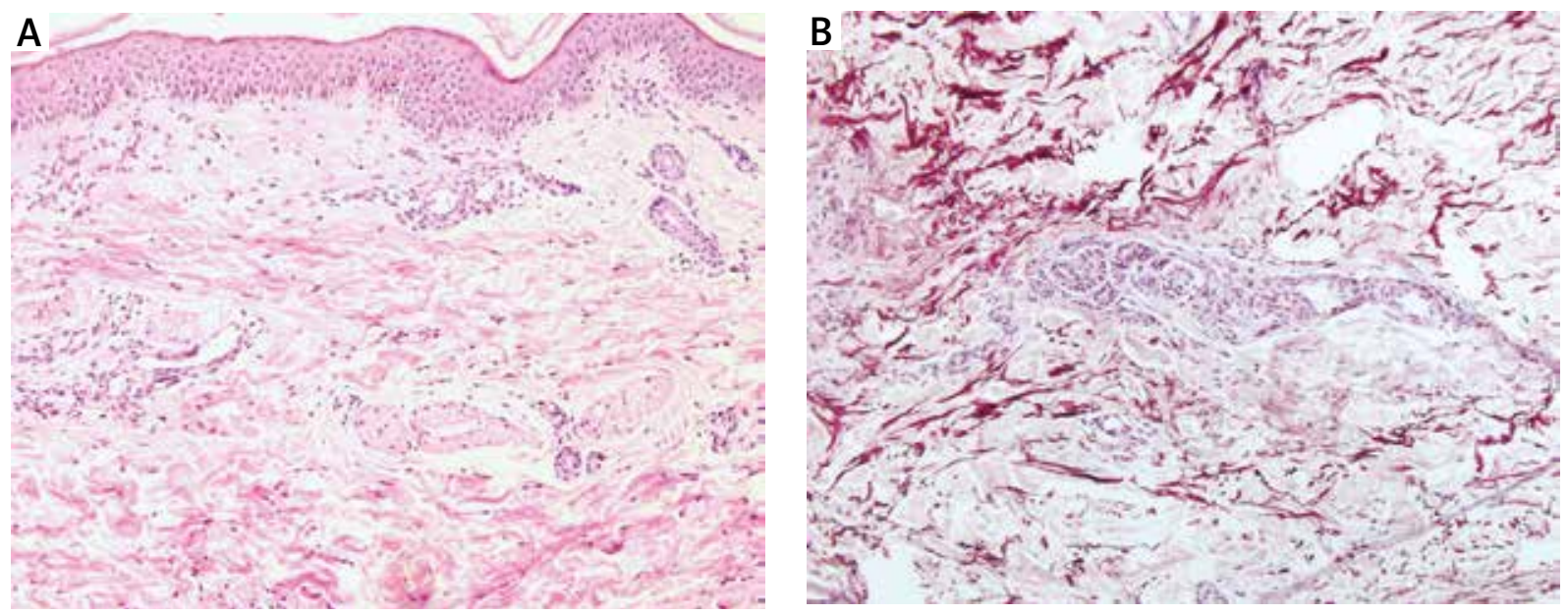

Figure 2. Histopathologic findings $20 \times$ magnification (A) hematoxylin eosin (B) orcein: the replacement of normal dermis and hypodermis structures by abnormal collagen. The vessels of the hypodermis show a thickened wall and decreased lumen size with mild inflammatory infiltrate

ous specialist consultations in the early life, the patient was finally diagnosed as localized scleroderma at age of 7 .

While in systemic sclerosis certain serum changes (such as the presence of Scl-70) are considered markers of the disease, laboratory changes are variable in localized scleroderma and their relationship to the underlying disease is questionable [10]. In our patient the antinuclear antibodies were negative. The antinuclear antibodies are present in $47-67 \%$ of patients with linear scleroderma, and anti-Scl70 antibodies, a marker of systemic sclerosis, are positive in $2-3 \%$ of patients with localized scleroderma. Anti-dsDNA antibodies are rarely observed in localized scleroderma. Eosinophilia and polyclonal hypergammaglobulinemia have been reported during the active stages of disease $[16,17]$.

Histology of scleroderma depends on two factors: the stage of the disease and the depth to which the disease extends. Scleroderma lesions are characterized by an initial inflammatory stage that is followed by a fibrosis stage and results in the replacement of normal dermis and hypodermis structures by abnormal collagen [2]. Diagnosis is essentially established by clinical examination supported by the skin biopsy. The disease is slowly progressing and spontaneous remission can occur.

Scleroderma and fibrous hamartoma of infancy belong to the wide spectrum of fibrous proliferation disorders and may share some of the etiopathogenetic pathways.

\section{Conflict of interest}

The authors declare no conflict of interest.

\section{References}

1. Takehara K, Sato S. Localized scleroderma is an autoimmune disorder. Rheumatology (Oxford) 2005; 44: 274-9.
2. Fett N, Werth VP. Update on morphea: part I. Epidemiology, clinical presentation, and pathogenesis. J Am Acad Dermatol 2011; 64: $217-28$

3. Herrick AL, Ennis H, Bhushan M, et al. Incidence of childhood linear scleroderma and systemic sclerosis in the UK and Ireland. Arthritis Care Res (Hoboken) 2010; 62: 213-8.

4. Zulian F. Systemic sclerosis and localized scleroderma in childhood. Rheum Dis Clin North Am 2008; 34: 239-55.

5. Miller MT, Sloane H, Goldberg MF, et al. Progressive hemifacial atrophy (Parry-Romberg disease). J Pediatr Ophthalmol Strabismus 1987; 24: 27-36.

6. Zulian F. Systemic manifestations in localized scleroderma. Curr Rheumatol Rep 2004; 6: 417-24.

7. Zulian F, Athreya BH, Laxer R, et al. Juvenile localized scleroderma: clinical and epidemiological features in 750 children. An international study. Rheumatology (Oxford) 2006; 45: 614-20.

8. Herrick AL, Ennis H, Bhushan M, et al. Clinical features of childhood localized scleroderma in an incidence cohort. Rheumatology (Oxford) 2011; 50: 1865-8.

9. Marzano AV, Menni S, Parodi A, et al. Localized scleroderma in adults and children. Clinical and laboratory investigations on 239 cases. Eur J Dermatol 2003; 13: 171-6.

10. Careta MF, Romiti R. Localized scleroderma: clinical spectrum and therapeutic update. An Bras Dermatol 2015; 90: 62-73.

11. Vinayak RS, Kumar S, Chandana S, et al. Fibrous hamartoma of infancy. Indian Dermatol Online J 2011; 2: 25-7.

12. Scott DM, Peńa JR, Omura EF. Fibrous hamartoma of infancy. J Am Acad Dermatol 1999; 41: 857-9.

13. Zulian F, Vallongo C, de Oliveira SK, et al. Congenital localized scleroderma. J Pediatr 2006; 149: 248-51.

14. Joshi A, Al-Mutairi N, Nour-Eldin O. Congenital skin lesions presenting as morphea in a 4-year-old. Pediatr Dermatol 2006; 23 : 94-5.

15. Castańón CS, Martínez Blanco J, Rodríguez Díaz E. Congenital morphea. An Pediatr (Barc) 2010; 73: 64-65.

16. Lipson J, O’Toole A, Kapur S. Delay in diagnosis of congenital linear scleroderma until adulthood. J Cutan Med Surg 2015; 19: 156-8.

17. Torok KS. Pediatric scleroderma: systemic or localized forms. Pediatr Clin North Am 2012; 59: 381-405. 